\title{
Piperine protects against myocardial ischemia/reperfusion injury by activating the PI3K/AKT signaling pathway
}

\author{
YUN-PENG LI ${ }^{1}$, ZHEN CHEN ${ }^{2}$ and YU-HUA CAI ${ }^{3}$ \\ ${ }^{1}$ Department of Cardiovasology, Affiliated Dongfeng Hospital, Hubei University of Medicine, Shiyan, Hubei 442008; \\ ${ }^{2}$ Department of Emergency and Evidence-Based Medicine, The Central Hospital of Wuhan, \\ Tongji Medical College, Huazhong University of Science and Technology, Wuhan, Hubei 430014; \\ ${ }^{3}$ Department of Cardiovasology, Jingzhou First Municipal Hospital, Jingzhou, Hubei 434000, P.R. China
}

Received August 8, 2019; Accepted March 18, 2020

DOI: $10.3892 /$ etm.2021.9805

\begin{abstract}
Piperine (PIP) exerts numerous pharmacological effects and its involvement in endoplasmic reticulum (ER) stress (ERS)-led apoptosis has garnered attention. The present study focused on whether PIP played protective effects on hypoxia/reoxygenation (H/R)-induced cardiomyocytes by repressing ERS-led apoptosis. The potential molecular mechanisms in association with the PI3K/AKT signaling pathway were investigated. Primary neonatal rat cardiomyocytes (NRCMs) were isolated and randomized into four groups: Control + vehicle group, control + PIP group, H/R + vehicle group and H/R + PIP group. The H/R injury model was constructed by $4 \mathrm{~h}$ of hypoxia induction followed by $6 \mathrm{~h}$ of reoxygenation. A total of $10 \mu \mathrm{M}$ PI3K/AKT inhibitor LY294002 was supplemented to the cells during the experiments. Cell viability and myocardial enzymes were detected to evaluate myocardial damage. A flow cytometry assay was performed to assess apoptotic response. Western blot analysis was performed to detect the expression of related proteins including PI3K, AKT, CHOP, GRP78 and cleaved caspase-12. The results showed that H/R markedly promoted myocardial damage as shown by the increased release of lactate dehydrogenase and creatine kinase levels, but a reduction in cell viability. In addition, ERS-induced apoptosis was markedly promoted by $\mathrm{H} / \mathrm{R}$ in NRCMs, as shown by the increased apoptotic rates and expression of C/EBP-homologous protein, endoplasmic reticulum chaperone $\mathrm{BiP}$ and caspase-12. PIP administration reversed cell injury and ERS-induced apoptosis in H/R. Mechanistic studies concluded that the apoptosis-inhibitory contributions and cardio-favorable effects of PIP were caused partly by the activation of the PI3K/AKT signaling pathway,
\end{abstract}

Correspondence to: Dr Yu-Hua Cai, Department of Cardiovasology, Jingzhou First Municipal Hospital, 8 Aviation Road, Jingzhou, Hubei 434000, P.R. China

E-mail: cardiology557@163.com

Key words: piperine, hypoxia-reoxygenation; apoptosis, endoplasmic reticulum stress, PI3K/AKT which was verified by LY294002 administration. To conclude, PIP can reduce ERS-induced apoptosis by activating the PI3K/AKT signaling pathway during the process of $H / R$ injury, which could be a potential therapeutic target for the treatment of myocardial ischemia/reperfusion injury.

\section{Introduction}

Acute myocardial infarction (AMI) remains one of the leading causes of mortality and disability worldwide $(1,2)$. Currently, the most effective way to rescue dying cardiomyocytes induced by AMI is timely revascularization (3). However, re-establishing blood flow to the ischemic area yields additional myocardial damage, which is termed as myocardial ischemia/reperfusion (I/R) injury (MIRI) (3). Although effective strategies such as percutaneous coronary intervention, intravenous thrombolysis and coronary artery bypass grafting timely limits infarct expansion and decrease in-hospital mortality, the subsequent occurrence of chronic heart failure and cardiac remodeling has gradually increased (1-3). Thus, MIRI affects the clinical efficacy of revascularization strategies, and is an important factor that causes the deterioration of myocardial function and electrophysiological status (1-4). Novel approaches for attenuating MIRI need to be explored in order for the maximal benefits of myocardial revascularization under AMI to be achieved.

Conventionally, the endoplasmic reticulum (ER) is involved in the biosynthesis, enfoldment, processing, excretion and transportation of proteins $(3,5,6)$. However, the disruption of ER homeostasis caused by MIRI, which is referred to as ER stress (ERS), leads to inaccurate synthesis and assembly of proteins $(3,5,6)$. Increasing evidence suggested that continuous activation of ERS and the consequent elevation of apoptotic cascade events act as crucial pathogenic mechanisms in MIRI $(3,5,6)$. Despite the confirmed benefits of ERS-induced apoptosis against MIRI, the molecular mechanisms of MIRI and its related therapeutic approaches remain to be elucidated $(3,5,6)$. Targeting ERS-related apoptosis may be a possible therapeutic approach for MIRI.

Piperine (PIP) is a phenolic substance found in black and long pepper that exerts multiple pharmacological effects, such as liver protection and tumor inhibition $(7,8)$. In recent 
years, the protective effect of PIP in I/R injury has received much attention $(9,10)$. Vaibhav et al $(10)$ found that piperine treatment inhibited I/R-induced neuronal dysfunction and that its protective function is achieved by reducing inflammation. Zou et al (9) also verified the neuroprotective effects of PIP in response to cerebral $I / R$ injury, the underlying mechanism of which is strictly related to the regulation of complement and coagulation cascades. However, the exact effects of PIP in MIRI are not clear and the possible pathological mechanisms associated with ERS-related apoptosis remains to be elucidated.

Accumulating data have proven that activation of the PI3K/AKT signaling pathway acts as a crucial molecular mechanism by which PIP exerts pleiotropic contributions under various pathological conditions (11-13). The activity of the PI3K/AKT pathway has multiple consequences in response to MIRI; in particular, it has anti-apoptotic effects against I/R injury related to ERS $(3,5,6)$. Further studies to investigate whether the anti-apoptotic effects produced by PIP in a PI3K/AKT-related manner play a role in cardioprotection during MIRI are required. The present study showed that PIP administration repressesed myocardial apoptosis and ERS in injury caused by $4 \mathrm{~h}$ of hypoxia followed by $6 \mathrm{~h}$ of reoxygenation $(\mathrm{H} / \mathrm{R})$, and that this may be related to activation of the PI3K/AKT pathway. Thus, the present study provided novel insights into the benefits and potential mechanisms of PIP against MIRI, and may offer a novel therapeutic target for MIRI treatment.

\section{Materials and methods}

Chemicals andreagents.PIP was purchasedfrom Sigma-Aldrich; Merch KGaA. The purity of PIP, which was $>98 \%$, was tested using high-performance liquid chromatography as previously described (12). DMEM, PBS, trypsin, FBS and collagenase type II were purchased from Gibco; Thermo Fisher Scientific, Inc. The CCK-8 assay and PI3K/AKT inhibitor LY294002 were purchased from Dojindo Molecular Technologies, Inc. and Selleck Chemicals, respectively. Lactate dehydrogenase (LDH) and creatine-kinase (CK) were detected using commercially available ELISA kits (Nanjing Jiancheng Bioengineering Institute; cat. A020-2-2 for LDH; cat. A032-1-1 for CK). The following primary antibodies were purchased from Abcam: Phosphorylated (p)-PI3K (1:600; cat. no. ab32509), PI3K (1:400; cat. no. ab191606), p-AKT (1:1,000; cat. no. ab182651), endoplasmic reticulum chaperone BiP (GRP78; 1:500; cat. no. ab21685), cleaved caspase-12 (1:600; cat. no. ab62484) and GAPDH (1:1,000; cat. no. ab181602). Antibodies against AKT (1:600; cat. no. 9272) and C/EBP-homologous protein (CHOP; 1:600; cat. no. 2895) were purchased from Cell Signaling Technology, Inc. Horseradish peroxidase-conjugated rabbit anti-rat immunoglobulin $\mathrm{G}$ secondary antibody was purchased from BIOSS. The BCA Protein Assay kit was purchased from Pierce; Thermo Fisher Scientific, Inc.

Neonatal rat cardiomyocyte (NRCM) culture. NRCMs were isolated from the ventricles of 1-3-day-old Sprague-Dawley rats as previously described (14). Animals were bred in a standard environment with controlled temperature $\left(20-25^{\circ} \mathrm{C}\right)$, humidity (40-60\%) and light condition (12 h light/dark cycle). The experiments and all animal care outlined in the present study were performed in adherence with the Guide for the Care and Use of Laboratory Animals published by the National Institutes of Health (NIH Publication, 8th Edition, 2011) $(1,2)$, and were approved by the Institutional Animal Care and Use Committee from the Central Hospital of Wuhan, Tongji Medical College, Huazhong University of Science and Technology. For cardiomyocyte isolation, all rats were sacrificed by decapitation. Subsequently, the hearts were quickly removed and large blood vessels were carefully excised. The obtained ventricles were rinsed in ice-cold PBS three times to remove residuary blood and placed in a dish with ice-cold PBS. Heart tissues were digested with $0.08 \%$ collagenase type II and $0.125 \%$ trypsin at room temperature for $5 \mathrm{~min}$. NRCMs were then centrifuged at $3,000 \mathrm{xg}$ for $10 \mathrm{~min}$ at $37^{\circ} \mathrm{C}$ and resuspended in DMEM containing $10 \%$ FBS and $1 \%$ penicillin/streptomycin (HyClone; GE Healthcare Life Sciences) in a humidified incubator at $37^{\circ} \mathrm{C}$ with $5 \% \mathrm{CO}_{2}$ and $95 \% \mathrm{O}_{2}$.

Construction of a H/R injury model and experimental groups. $\mathrm{H} / \mathrm{R}$ injury was established as previously described with minor modifications (15). Cultured NRCMs were washed twice with PBS and preserved in serum-free DMEM. Subsequently, the cells were incubated at $37^{\circ} \mathrm{C}$ in an anaerobic chamber for $4 \mathrm{~h}$. The cells were then moved into a normal incubator with $95 \% \mathrm{O}_{2}$ and $5 \% \mathrm{CO}_{2}$ at $37^{\circ} \mathrm{C}$ for an additional $6 \mathrm{~h}$ for reoxygenation.

To determine the possible function of PIP in MIRI, different dosage gradients of PIP $(1,5,10$ and $20 \mu \mathrm{M})$ were administered to first measure the most effective dose against MIRI. A total of 16 neonatal rats were used in each group ( $n=4$ per group). Another experiment was then designed to explore the precise roles and molecular mechanisms of PIP in response to H/R. Primary cardiomyocytes were randomly divided into four groups: The control + vehicle group, which consisted of NRCMs cultured in normoxic conditions without PIP treatment; the control + PIP group, which consisted of NRCMs cultured in normoxic conditions with PIP treatment; the H/R + vehicle group, which consisted of NRCMs cultured in H/R conditions without PIP treatment and the H/R + PIP group, which consisted of NRCMs cultured in H/R conditions with PIP treatment. During this experiment, 20 neonatal rats were used for each group ( $n=5$ per group). Additionally, to detect the underlying mechanism of the association with the PI3K/AKT pathway, cells were pre-treated with $10 \mu \mathrm{M}$ LY294002 for 30 min prior to PIP administration, and were then subjected to $\mathrm{H} / \mathrm{R}$ intervention. During this experiment, 24 neonatal rats were used for each group ( $\mathrm{n}=6$ per group).

Cell viability assay. The CCK- 8 assay was used to measure cell viability in each group as previously described (16). The optical density values were detected at a wavelength of $450 \mathrm{~nm}$ using a microplate reader (Bio-Rad Laboratories, Hercules, CA). Cell viability was evaluated as a percentage relative to the control group.

Measurement of markers of myocardial injury. After the indicated procedures, the medium in each group was collected using a sterile pipette. Commercially available biochemical kits were used in line with the manufacturer's instructions to detect LDH and CK. 

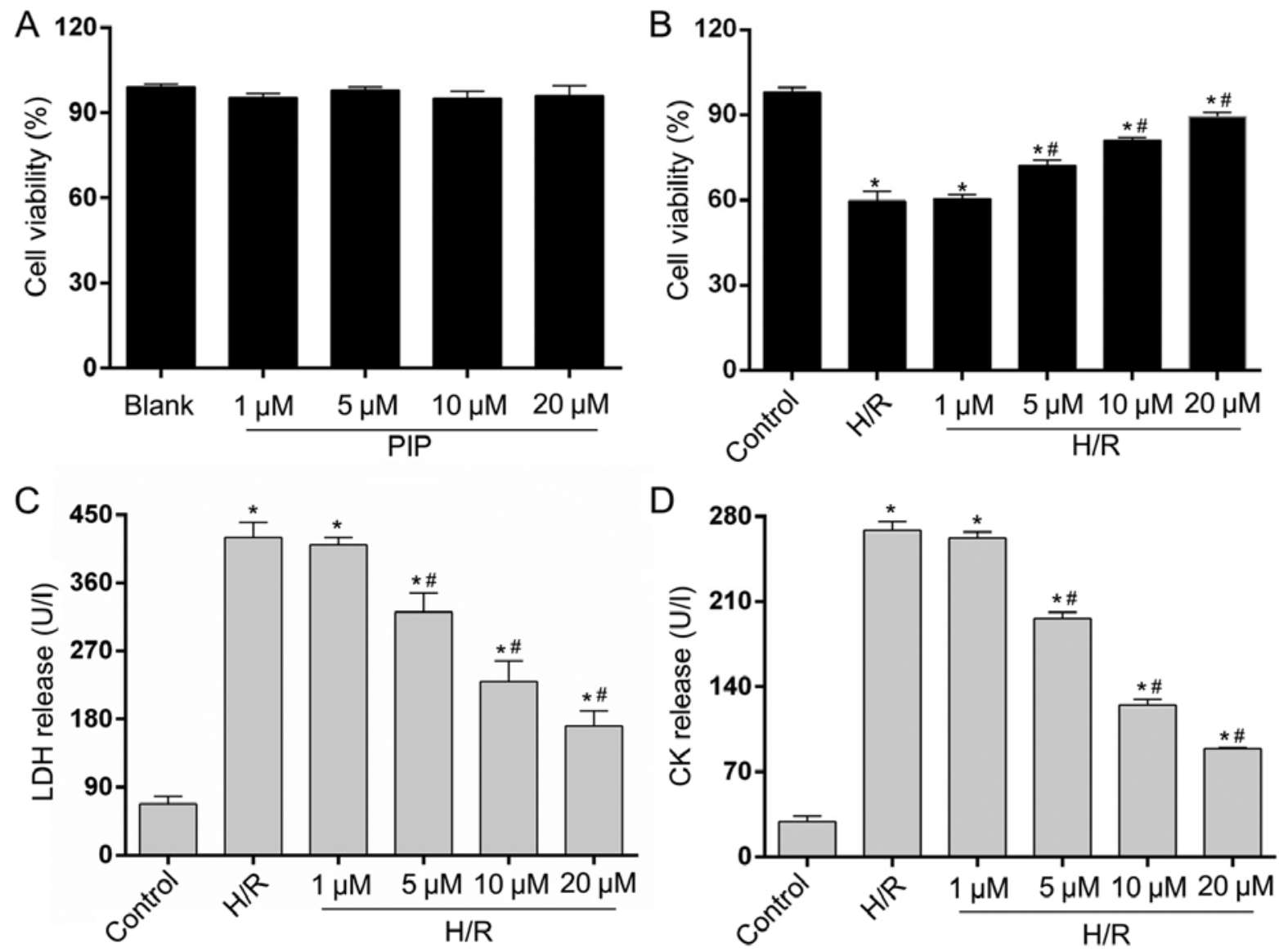

Figure 1. PIP attenuates H/R-induced NRCM injury. (A) The cytotoxicity of PIP $(1,5,10$, and $20 \mu \mathrm{M})$ on NRCM viability assessed by a CCK-8 assay. The effect of PIP on (B) cell viability, (C) LDH release and (D) CK release following H/R insult. $n=4$. ${ }^{*} \mathrm{P}<0.05$ vs control; and ${ }^{~} \mathrm{P}<0.05$ vs. the $\mathrm{H} / \mathrm{R}$ group. PIP, piperine; NRCM, neonatal rat cardiomyocyte; H/R, hypoxia/reoxygenation; LDH, lactate dehydrogenase; CK, creatine-kinase.

Apoptosis detection. Apoptosis of NRCMs was measured by a flow cytometry assay as previously described $(16,17)$. Cells were maintained in $100 \mu 1$ of binding buffer, which included $5 \mu \mathrm{l}$ Annexin V-APC and $5 \mu \mathrm{l}$ 7-aminoactinomycin D. Stained cells were observed using a flow cytometer (Beckman Coulter), followed by the fluorescence-activated cell sorting on a flow cytometeric assay (CytoFLEX; Beckman Coulter, Inc., Brea, CA, USA). NRCMs positive for 7-AAD or Annexin V-APC were recognized as apoptotic cells.

Western blotting. Western blotting was used to detect protein expression as previously described $(3,16)$. Myocardial samples were homogenized and lysed in RIPA buffer (Beyotime Institute of Biotechnology) at a mass/volume ratio of $100 \mathrm{mg} / \mathrm{ml}$ Protein was then extracted and protein concentration was detected using a BCA kit (Beyotime Institute of Biotechnology). Then, $10 \%$ SDS-PAGE was performed to separate the proteins, and $50 \mu \mathrm{g}$ of extracted proteins in each group were electrophoretically transferred onto PVDF membranes (EMD Millipore). Next, the membranes were blocked by $5 \%$ skimmed milk in TBST for $2 \mathrm{~h}$ at $37^{\circ} \mathrm{C}$. Subsequently the membranes were incubated with antibodies against CHOP, GRP78, caspase-12, PI3K, p-PI3K, AKT and p-AKT overnight at $4{ }^{\circ} \mathrm{C}$. GAPDH was used as the internal reference protein. The horseradish peroxidase-conjugated secondary antibodies were added for another $2 \mathrm{~h}$ at $37^{\circ} \mathrm{C}$. The protein bands were visualized using an ECL kit (Thermo Fisher Scientific, Inc.).
Reverse transcription-quantitative PCR (RT-qPCR). RT-qPCR was performed to detect mRNA levels as previously described $(3,16)$. Total RNA was extracted from NRCMs using TRIzol ${ }^{\circledR}$ reagent (Invitrogen; Thermo Fisher Scientific, Inc.) according to the manufacturers protocol, after the indicated treatments. qPCR was performed using a SYBR-Green Master Mix kit (cat. no. 4472918; Thermo Fisher Scientific, Inc.) on a 7500 ABI Prism system (Applied Biosystems). The following thermocycling conditions were used for the qPCR: Initial denaturing at $50^{\circ} \mathrm{C}$ for $2 \mathrm{~min}, 40$ cycles of denaturation at $95^{\circ} \mathrm{C}$ for $30 \mathrm{sec}$ and final extension at $60^{\circ} \mathrm{C}$ for $30 \mathrm{sec}$. The mRNA expression levels of CHOP, GRP78 and caspase-12 were normalized to GAPDH levels. The fold-change of the mRNA levels relative to the control cells was calculated using the $2^{-\Delta \Delta C q}$ method (3-5). The following primer pairs were used for the qPCR: CHOP forward, 5'-TAGCTTGGCTGACTG AGGAGC-3' and reverse, 5'-CTTCAGCAAGCTGTGCCA CT-3'; GRP78 forward, 5'-GATAATCAGCCCACCGTAACA AT-3' and reverse, 5'-GCAAACTTCTCGGCGTCATT-3' and caspase-12 forward, 5'-CATTGCCAATTCCGACAAAC-3' and reverse, 5'-CCTTCCTTCTCCATCACTGGA-3'; GAPDH forward, 5'-ACAGCAACAGGGTGGTGGAC-3' and reverse, 5'-TTTGAGGGTGCAGCGAACTT-3'.

Statistical analysis. SPSS 17.0 (SPSS, Inc.) was used for statistical analysis. Data were expressed as the mean \pm SD. The Student's t-test was used for comparisons within groups. 

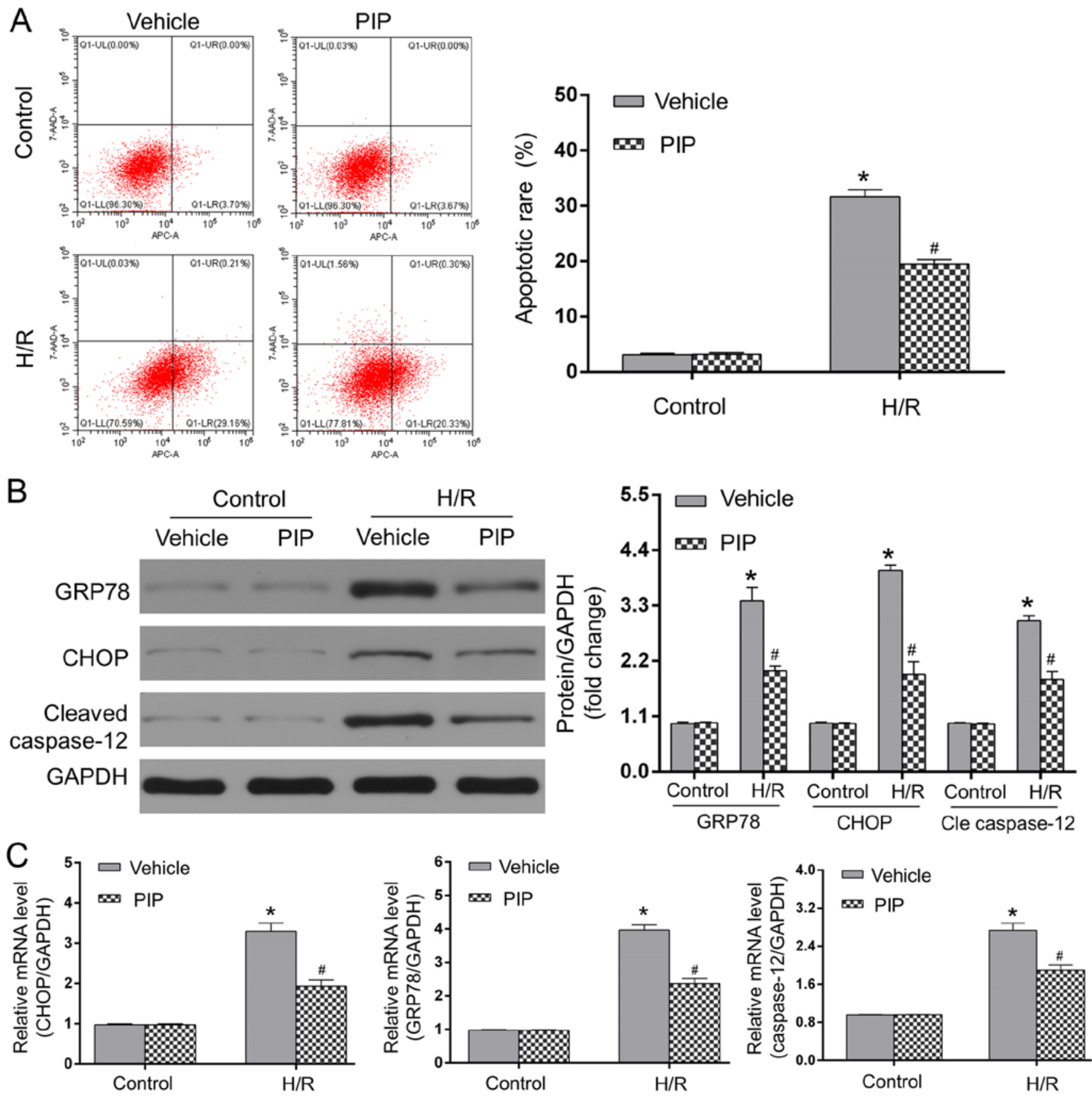

Figure 2. PIP treatment alleviates ERS-induced apoptosis during H/R injury. (A) Apoptotic cells were monitored by flow cytometry. (B) Protein levels of GRP78, CHOP and caspase-12 were detected by western blotting. The left panel shows representative blots and the right panel shows quantitative results of western blotting. (C) mRNA levels of GRP78, CHOP and caspase- 12 were detected by reverse transcription-quantitative PCR. $n=4$. " $\mathrm{P}<0.05$ vs. the vehicle + control group; ${ }^{\prime} \mathrm{P}<0.05$ vs. the vehicle $+\mathrm{H} / \mathrm{R}$ group. PIP, piperine; ERS, endoplasmic reticulum stress; $\mathrm{H} / \mathrm{R}$, hypoxia/reoxygenation; GRP78, endoplasmic reticulum chaperone BiP; CHOP, C/EBP-homologous protein; Cle caspase-12, cleaved caspase-12.

The statistical comparisons among multiple groups were performed using analysis of variance (ANOVA) followed by a post hoc Tukey's test. $\mathrm{P}<0.05$ was considered to indicate a statistically significant difference.

\section{Results}

PIP attenuates H/R-induced NRCM injury. A CCK-8 assay was performed to assess cell viability (17). The four different PIP concentrations $(1,5,10$ and $20 \mu \mathrm{M})$ had no effect on cell viability and exhibited very low cytotoxicity in control NRCMs (Fig. 1A). To evaluate the possible effects of PIP on H/R injury,
NRCMs underwent $4 \mathrm{~h}$ of hypoxia and $6 \mathrm{~h}$ of reoxygenation. As shown in Fig. 1B, cell viability was reduced following H/R insult compared with controls. In addition, the various doses of PIP $(5,10$ and $20 \mu \mathrm{M})$ increased cell viability compared with H/R-induced cells. Cell viability significantly increased following treatment with 5 and $10 \mu \mathrm{M}$ PIP, and the highest improvement in cell viability compared with the H/R group was obtained following treatment with $20 \mu \mathrm{M}$ PIP. LDH and CK release were detected to assess NRCM death. The release of LDH (Fig. 1C) and CK (Fig. 1D) caused by H/R injury was limited starting at a concentration of $5 \mu \mathrm{M}$. Minimal levels of $\mathrm{LDH}$ and CK were measured in cells treated with $20 \mu \mathrm{M}$ PIP 

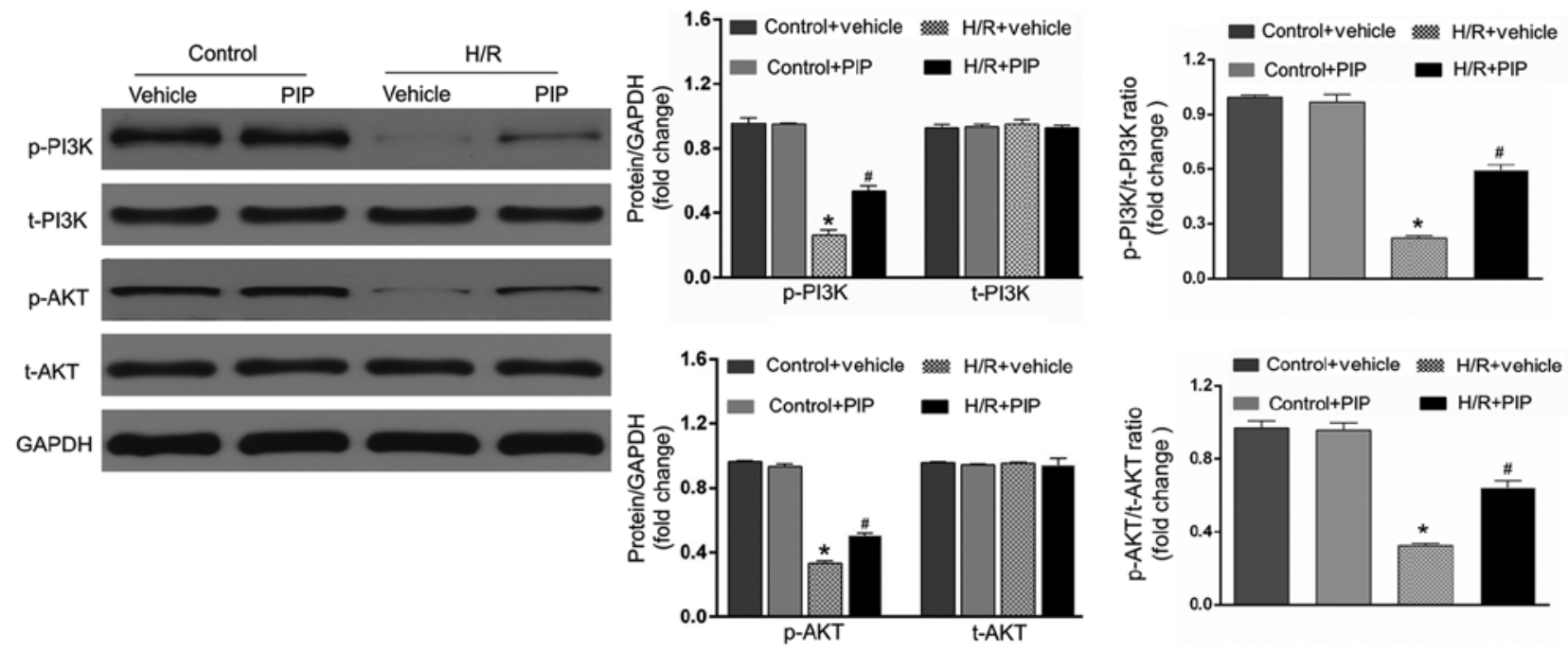

Figure 3. PIP pretreatment activates the PI3K/AKT signaling pathway. The left panel shows representative western blots of p-PI3K, PI3K, p-Akt and Akt. The right panel shows corresponding bar graphs of densitometric analysis in neonatal rat cardiomyocytes detected by western blotting. $n=4$. "P<0.05 vs. the vehicle + control group and ${ }^{\#} \mathrm{P}<0.05$ vs. the vehicle $+\mathrm{H} / \mathrm{R}$ group. PIP, piperine; p-PI3K, phosho-PI3K; t-PI3K, total PI3K; p-AKT, phospho-AKT; t-AKT, total AKT; $\mathrm{H} / \mathrm{R}$; hypoxia/reoxygenation.

compared with other concentrations. No statistical difference in $\mathrm{LDH}$ and $\mathrm{CK}$ release or cell viability was found between the $1 \mu \mathrm{M}$ PIP-treated and $\mathrm{H} / \mathrm{R}$ groups. A total of $20 \mu \mathrm{M}$ PIP was used for subsequent experiments. The results suggested that PIP could increase cell survival and repress cell damage in NRCMs under H/R injury.

PIP treatment alleviates ERS-induced apoptosis during $H / R$ injury. Myocardial $\mathrm{H} / \mathrm{R}$ injury is related to the activation of ERS-induced apoptosis and PIP was indicated to be involved in ERS progression $(3,18,19)$. As an ER chaperone, GRP78 is crucial for myocardial apoptosis during $\mathrm{H} / \mathrm{R}$, particularly since ERS can drive the apoptotic cascade, including the activation of caspase-12 in a CHOP-dependent manner (3). To determine whether $20 \mu \mathrm{M}$ PIP affects H/R injury in an ERS-related manner, the protein and mRNA expressions of GRP78, CHOP and caspase-12 in apoptotic NRCMs were assessed. As shown in Fig. 2A, minimal apoptotic rates were observed in the control + vehicle and control + PIP groups, whereas compared to the controls, H/R injury significantly increased cardiomyocyte apoptosis. PIP treatment significantly decreased the number of apoptotic cells under $\mathrm{H} / \mathrm{R}$ conditions compared with vehicle-treated cells following $\mathrm{H} / \mathrm{R}(\mathrm{P}<0.05)$. The expression of ERS-related proteins, CHOP, GRP78 and caspase-12 in each group was detected using western blotting and RT-qPCR. As shown in Fig. 2B and C, H/R injury elevated protein and mRNA expressions of GRP78, CHOP and caspase-12 compared with the control groups $(\mathrm{P}<0.05)$. However, PIP administration significantly decreased the levels of these proteins and mRNAs in response to $\mathrm{H} / \mathrm{R}$ injury compared with those in the $\mathrm{H} / \mathrm{R}+$ vehicle group $(\mathrm{P}<0.05)$. Therefore, the above data indicated that PIP mitigated H/R-led ERS and subsequent apoptosis in NRCMs.

PIP activates the PI3K/AKT signaling pathway. To further investigate the underlying mechanisms behind PIP in alleviating $\mathrm{I} / \mathrm{R}$ injury, the expression of genes involved in the PI3K/AKT signaling pathway was determined. As shown in Fig. 3, H/R significantly downregulated the expression of $\mathrm{p}-\mathrm{PI} 3 \mathrm{~K}$ and p-AKT compared with the control group $(\mathrm{P}<0.05)$. However, pretreatment with PIP at the onset of $\mathrm{H} / \mathrm{R}$ increased the expression of p-PI3K and p-AKT compared with the H/R + vehicle group. No significant difference in the expression of PI3K and AKT was observed between the different groups. Therefore, it can be speculated that H/R injury is ameliorated by PIP, possibly through activating the PI3K/AKT signaling pathway.

PI3K/AKT repression reversed the inhibitory effects of PIP on $H / R$ injury. To further validate whether the PI3K/AKT pathway served as a causative mediator of PIP-induced repression of $\mathrm{H} / \mathrm{R}$, NRCMs were pre-administrated with the specific PI3K/AKT inhibitor LY294002 and subjected to H/R injury. The results showed that LY294002 rescued H/R-induced cell death and ERS-related apoptosis, as demonstrated by the restored LDH and CK activity (Fig. 4B), apoptotic rate (Fig. 4C), restored GRP78, CHOP and caspase-12 mRNA levels (Fig. 4D) and suppression of cell viability (Fig. 4A). PIP lost the ability to reverse these alternations in the presence of LY294002 under H/R insult (Fig. 4A-D). Furthermore, in the presence of LY294002, there was no significant difference in these alterations with or without PIP administration. Thus, these findings indicated that PIP pretreatment alleviates ERS-induced apoptosis in H/R-exposed NRCMs mainly by activating the PI3K/AKT pathway.

\section{Discussion}

Previous studies found that PIP could alleviate pressure overload-led cardiac remodeling and neuronal I/R injury $(9,12)$, but its role in MIRI and the possible mechanisms in association with the PI3K/AKT pathway remain to be elucidated. The present study observed that PIP treatment reduced myocardial $\mathrm{H} / \mathrm{R}$ injury, which was characterized by the upregulation of cell activity and the decreased release of LDH and CK. In addition, PIP administration significantly alleviated myocardial 
A

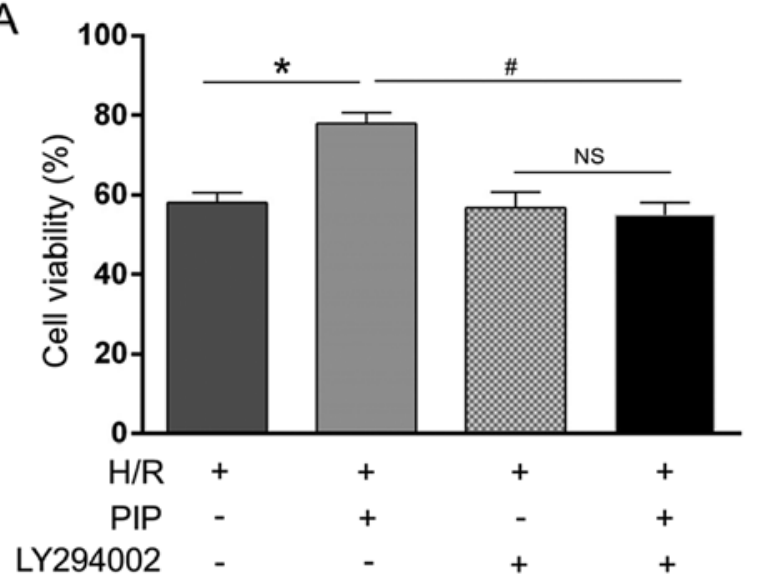

B

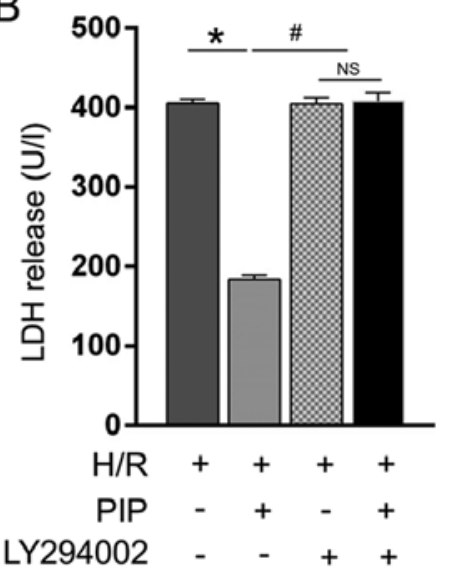

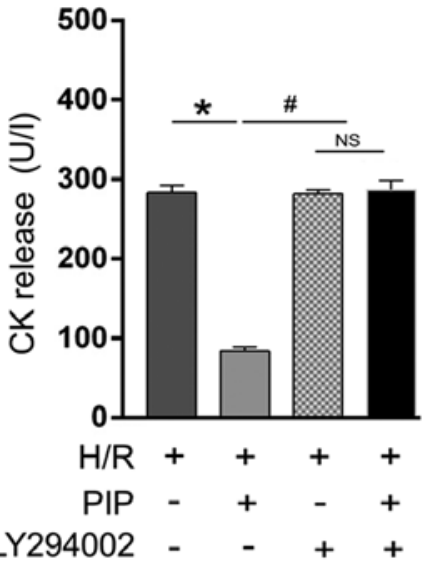

C
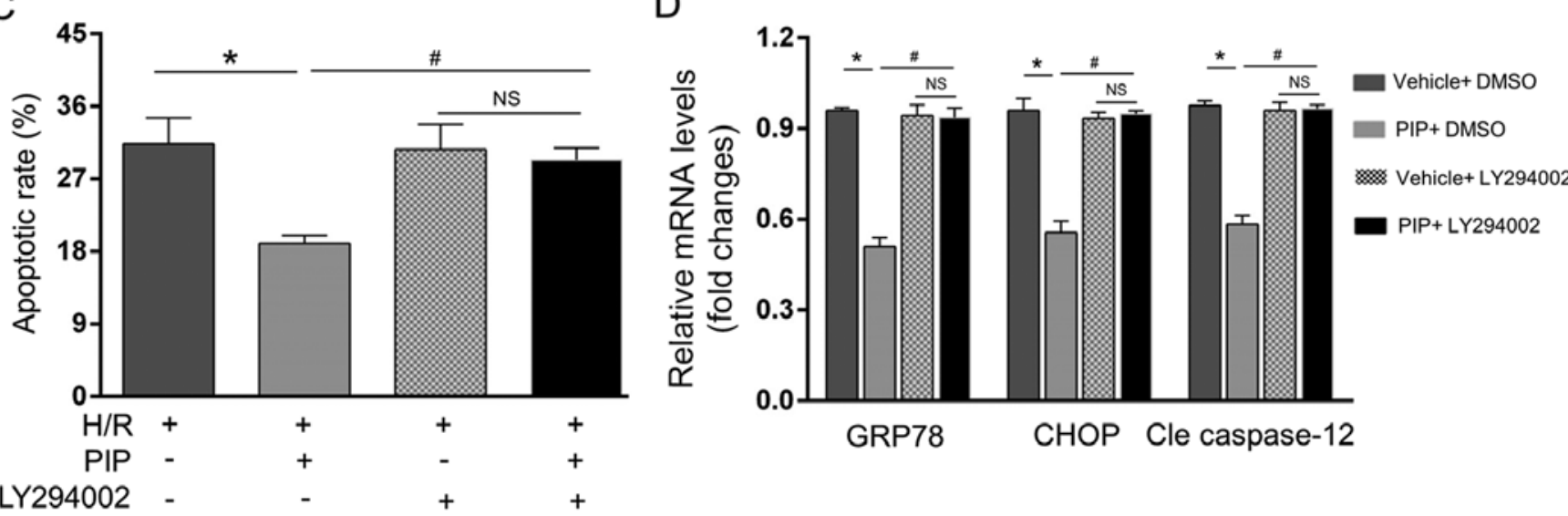

Figure 4. PI3K/AKT inhibition reverses the inhibitory effects of PIP on H/R. CCK-8 and ELISA assays were used to detect (A) cell viability and (B) LDH and CK release, respectively. Apoptosis was measured by (C) flow cytometry and (D) GRP78, CHOP and caspase-12 mRNA levels were detected by reverse transcription-quantitative PCR. $n=4$. " $\mathrm{P}<0.05$ vs. the $\mathrm{H} / \mathrm{R}$ group and ${ }^{*} \mathrm{P}<0.05$ vs. the $\mathrm{PIP}+\mathrm{H} / \mathrm{R}$ group. NS, not significant; PIP, piperine; H/R, hypoxia/reoxygenation; $\mathrm{LDH}$, lactate dehydrogenase; $\mathrm{CK}$, creatine-kinase; GRP78, endoplasmic reticulum chaperone BiP; CHOP, C/EBP-homologous protein; Cle caspase-12, cleaved caspase-12.

apoptosis and inhibited the protein expression of ERS-related molecules, such as GRP78, CHOP and caspase-12. Mechanistic studies observed that PIP markedly promoted the PI3K/AKT signaling pathway, while LY294002 reversed its protective function against H/R-induced damage and ERS-related apoptosis. The above results therefore indicated that PIP ameliorated H/R-induced and ERS-related apoptosis mainly via the PI3K/AKT-dependent pathway.

Evidence has suggested that PIP has pleiotropic impacts on a variety of pathological conditions, such as glutamate release from rat hippocampal nerve terminals, lupus nephritis, 6-hydroxydopamine-induced Parkinson's disease and prostate cancer development, possibly due to its multiple bioactivities (20-23). Recent findings revealed that PIP also seems to be beneficial in the treatment of cardiovascular disease. In a rat model of isoproterenol (ISO)-induced myocardial infarction, Dhivya et al (24) demonstrated that PIP pretreatment acted as a potent therapeutic agent with antioxidant and anti-dyslipidemic actions against ISO-caused myocardial ischemiaIn addition, Ma et al (12) indicated that pretreatment with PIP can attenuate pathological cardiac fibrosis following pressure overload and ISO injection. Latest research indicated that PIP exerts a pivotal effect on the course of the pathological process of I/R (9). For example, in a rat model of cerebral I/R injury, Zou et al (9) showed that PIP pretreatment protected neurocytes from I/R damage by the regulation of complement and coagulation cascades. Vaibhav et al (10) found that PIP administration rescued ischemic penumbral zone neurons via its anti-inflammatory properties, thereby suppressing ischemic cell death. However, to the best of our knowledge, the involvements and potential mechanisms of PIP in MIRI remain to be elucidated. A recent study has demonstrated that PIP is an important mediator in the initiation and development of ERS and subsequent apoptotic activation (18). Yaffe et al (25) demonstrated that PIP treatment inhibited the apoptosis of human colon cancer cells triggered by ERS. PIP was verified to regulate ERS progression and thus is a possible therapeutic agent that limited ERS-related renal apoptosis and cell death (19). In terms of the possible involvement of PIP in I/R injury and ERS-related apoptosis, it was hypothesized that PIP could exert protective roles on cardiomyocytes and play inhibitory roles in ERS-related apoptosis in response to $H / R$ insult. Consequently, the present research provided evidence that PIP has an advantageous role in H/R-injured NRCMs and can also alleviate myocardial I/R disorder.

Timely and effective reperfusion of ischemic heart tissue was confirmed to be the most efficacious remedy for AMI (1-3). However, the accompanying I/R injury largely offsets this beneficial effect (1-3). Effective methods for mitigating the adverse influences of reperfusion still need to be further determined. 
Myocardial apoptosis is considered to be one of the most critical mechanisms of MIRI pathogenesis, and the ERS-elicited apoptotic cascade has drawn considerable attention $(3,5)$. Previous findings showed that MIRI could result in severe ERS as GRP78 levels are drastically enhanced following MIRI (3). GRP78 activation then causes a depolymerization reaction between ER-transmembrane transducers and GRP78, resulting in the activation of caspase-12 and apoptosis (3). CHOP is a specific transcription factor involved in ERS-induced apoptosis (3), and prolonged ERS promotes the expression of CHOP (3). The present study demonstrated that MIRI could cause significant myocardial damage and ERS. However, PIP administration reduced GRP78, CHOP and caspase-12 expression, ameliorating the parameters relative to the severity of the H/R injury. Therefore, PIP has the ability to protect cardiomyocytes mainly by mitigating ERS-related apoptosis.

Based on the above findings, the potential molecular mechanisms by which PIP exerts its protective effects against H/R injury and ERS-related apoptosis were further explored. PI3K and its downstream target serine/threonine kinase AKT belong to a conserved family of signal transduction enzymes $(3,5,6)$. Activation of the PI3K/AKT pathway is considered an endogenous regulatory mechanism that facilitates cell survival in response to harmful external stimuli $(3,5,6)$. Numerous studies provided evidence that the PI3K/AKT signaling pathway plays a critical role in the pathological process of MIRI $(3,5,6)$, and compromised ERS-related apoptosis was verified to be an important mechanism for the protective role of the PI3K/AKT activation $(3,5,6)$. Zhang et al (3) showed that the PI3K/AKT axis participated in anti-I/R effects via ERS repression, resulting in reduced GRP78, CHOP and caspase-12 expression and apoptotic inhibition. Deng et al (26) demonstrated that activation of the PI3K/AKT signaling pathway promoted myocardial survival by attenuating ERS-induced apoptosis. Moreover, there is a close relationship between PIP and the activation of the PI3K/AKT pathway (13). Chen et al (13) revealed that PIP promoted Leydig cell development but inhibited spermatogenesis in rats by activating AKT. Consistent with a previous study (13), the present study validated that pretreatment with PIP not only inhibited apoptosis induced by H/R injury, but also upregulated the expression of p-PI3K and p-AKT. To further verify the causal relationship between the activation of the PI3K/AKT signaling pathway and PIP administration, LY294002 was added to cells at the onset of H/R injury. The cardioprotective efficacy and inhibitory effects of PIP on ERS-related apoptosis were significantly reversed in the presence of LY294002. Therefore, the above data indicated that PIP administration can decrease ERS-associated apoptosis and H/R-induced cell damage in a PI3K/AKT-dependent manner. Of note, the LY294002 supplement at the onset of control group was missing in this experiment. This is a limitation of the manuscript that it would be beneficial to include this group to confirm the H/R-inhibitory roles of PIP. Furthermore, the specific roles of PIP in alterations in the PI3K/AKT pathway remain controversial. Ma et al (12) found that PIP attenuated cardiac fibrosis via the inhibition of AKT after pressure overload, inconsistent with previous reports elucidating that PIP repressed migration and proliferation potentially via a reduction in the AKT pathway in non-cardiomyocytes (11). These discrepancies might be partially explained by the fact that the actions of PIP on the activation of the PI3K/AKT pathway vary in a cell-type and stimulus-dependent manner.

In conclusion, results of the present study have demonstrated that pretreatment with PIP alleviated MIRI by attenuating ERS-associated apoptosis in a PI3K/AKT-dependent manner. Numerous other signaling pathways including the TLR4/NF- $\kappa \mathrm{B}$ pathway (16) and the LncRNA/miRNA pathway (2) are involved in the pathophysiological process of MIRI. Further studies are necessary to explore whether any other signaling pathways involved in the protective effect of PIP in MIRI. Taken together, the present results indicated that PIP might be a prospective therapeutic option for MIRI.

\section{Acknowledgements}

Not applicable.

\section{Funding}

No funding was received.

\section{Availability of data and materials}

The datasets used and/or analyzed during the current study are available from the corresponding author on reasonable request.

\section{Authors' contributions}

YPL and YHC conceived and designed the experiments. ZC and YPL performed majority of the experiments, collected the data and finished the statistical analysis. All authors contributed to manuscript preparation and revision. All authors read and approved the final manuscript.

\section{Ethics approval and consent to participate}

Not applicable.

\section{Patient consent for publication}

Not applicable.

\section{Competing interests}

The authors declare that they have no competing interests.

\section{References}

1. Zeng G,Lian C, Yang P,Zheng M, Ren Hand Wang H: E3-ubiquitin ligase TRIM6 aggravates myocardial ischemia/reperfusion injury via promoting STAT1-dependent cardiomyocyte apoptosis. Aging (Albany NY) 11: 3536-3550, 2019.

2. Ruan Z, Wang S, Yu W and Deng F: LncRNA MALAT1 aggravates inflammation response through regulating PTGS2 by targeting miR-26b in myocardial ischemia-reperfusion injury. Int J Cardiol 288: 122, 2019.

3. Zhang BF, Jiang H, Chen J, Guo X, Li Y, Hu Q and Yang S: Nobiletin ameliorates myocardial ischemia and reperfusion injury by attenuating endoplasmic reticulum stress-associated apoptosis through regulation of the PI3K/AKT signal pathway. Int Immunopharmacol 73: 98-107, 2019. 
4. Chang JC, Lien CF, Lee WS, Chang HR, Hsu YC, Luo YP, Jeng JR, Hsieh JC and Yang KT: Intermittent intermittent hypoxia prevents myocardial mitochondrial $\mathrm{Ca}^{2+}$ overload and cell death during ischemia/reperfusion: The role of reactive oxygen species. Cells 8: pii: E564, 2019.

5. Shu Z, Yang Y, Yang L, Jiang H, Yu X and Wang Y: Cardioprotective effects of dihydroquercetin against ischemia reperfusion injury by inhibiting oxidative stress and endoplasmic reticulum stress-induced apoptosis via the PI3K/Akt pathway. Food Funct 10: 203-215, 2019.

6. Shen D, Chen R, Zhang L, Rao Z, Ruan Y, Li L, Chu M and Zhang Y: Sulodexide attenuates endoplasmic reticulum stress induced by myocardial ischaemia/reperfusion by activating the PI3K/Akt pathway. J Cell Mol Med 23: 5063-5075, 2019.

7. Manayi A, Nabavi SM, Setzer WN and Jafari S: Piperine as a potential anti-cancer agent: A review on preclinical studies. Curr Med Chem 25: 4918-4928, 2018.

8. Abdel-Daim MM, Sayed AA, Abdeen A, Aleya L, Ali D, Alkahtane AA, Alarifi S and Alkahtani S: Piperine enhances the antioxidant and anti-inflammatory activities of thymoquinone against Microcystin-LR-induced hepatotoxicity and neurotoxicity in mice. Oxid Med Cell Longev 2019: 1309175, 2019.

9. Zou Y, Gong P, Zhao W, Zhang J, Wu X, Xin C, Xiong Z, Li Z, Wu X, Wan Q, et al: Quantitative iTRAQ-based proteomic analysis of piperine protected cerebral ischemia/reperfusion injury in rat brain. Neurochem Int 124: 51-61, 2019.

10. Vaibhav K, Shrivastava P, Javed H, Khan A, Ahmed ME, Tabassum R, Khan MM, Khuwaja G, Islam F, Siddiqui MS, et al: Piperine suppresses cerebral ischemia-reperfusion-induced inflammation through the repression of COX-2, NOS-2, and $\mathrm{NF}-\kappa \mathrm{B}$ in middle cerebral artery occlusion rat model. Mol Cell Biochem 367: 73-84, 2012.

11. Zeng Y and Yang Y: Piperine depresses the migration progression via downregulating the Akt/mTOR/MMP-9 signaling pathway in DU145 cells. Mol Med Rep 17: 6363-6370, 2018.

12. Ma ZG, Yuan YP, Zhang X, Xu SC, Wang SS and Tang QZ: Piperine attenuates pathological cardiac fibrosis via PPAR- $\gamma / \mathrm{AKT}$ pathways. Ebiomedicine 18: 179-187, 2017.

13. Chen X, Ge F, Liu J, Bao S, Chen Y, Li D, Li Y, Huang T, Chen X, Zhu Q, et al: Diverged effects of piperine on testicular development: Stimulating leydig cell development but inhibiting spermatogenesis in rats. Front Pharmacol 9: 244, 2018.

14. Hu S, Cheng M, Guo X, Wang S, Liu B, Jiang H, Huang C and Wu G: Down-regulation of miR-200c attenuates AngII-induced cardiac hypertrophy via targeting the MLCK-mediated pathway. J Cell Mol Med 23: 2505-2516, 2019.

15. Cheng F, Yuan W, Cao M, Chen R, Wu X and Yan J: Cyclophilin a protects cardiomyocytes against hypoxia/reoxygenation-induced apoptosis via the AKT/Nox2 pathway. Oxid Med Cell Longev 2019: 2717986, 2019.
16. Guo X, Jiang H, Chen J, Zhang BF, Hu Q, Yang S, Yang J and Zhang J: RP105 ameliorates hypoxia/reoxygenation injury in cardiac microvascular endothelial cells by suppressing TLR4/MAPKs/NF- $\kappa B$ signaling. Int J Mol Med 42: 505-513, 2018.

17. Sun Y, Liu L, Yuan J, Sun Q, Wang N and Wang Y: RP105 protects PC12 cells from oxygenglucose deprivation/reoxygenation injury via activation of the PI3K/AKT signaling pathway. Int $\mathrm{J}$ Mol Med 41: 3081-3089, 2018

18. Guo J, Cui Y, Liu Q, Yang Y, Li Y, Weng L, Tang B, Jin P, Li XJ, Yang S and Li S: Piperine ameliorates SCA17 neuropathology by reducing ER stress. Mol Neurodegener 13: 4, 2018.

19. Hammad AS, Ravindran S, Khalil A and Munusamy S: Structure-activity relationship of piperine and its synthetic amide analogs for therapeutic potential to prevent experimentally induced ER stress in vitro. Cell Stress Chaperones 22: 417-428, 2017.

20. Peng X, Yang T, Liu G, Liu H, Peng Y and He L: Piperine ameliorated lupus nephritis by targeting AMPK-mediated activation of NLRP3 inflammasome. Int Immunopharmacol 65: 448-457, 2018.

21. Hsieh TY, Chang Y and Wang SJ: Piperine-mediated suppression of voltage-dependent $\mathrm{Ca}^{2+}$ influx and glutamate release in rat hippocampal nerve terminals involves 5HT1A receptors and $\mathrm{G}$ protein $\beta \gamma$ activation. Food Funct 10: 2720-2728, 2019.

22. Singh $S$ and Kumar P: Piperine in combination with quercetin halt 6-OHDA induced neurodegeneration in experimental rats: Biochemical and neurochemical evidences. Neurosci Res 133: 38-47, 2018.

23. George K, Thomas NS and Malathi R: Piperine blocks voltage gated $\mathrm{K}^{+}$current and inhibits proliferation in androgen sensitive and insensitive human prostate cancer cell lines. Arch Biochem Biophys 667: 36-48, 2019.

24. Dhivya V, Priya LB, Chirayil HT, Sathiskumar S, Huang CY and Padma VV: Piperine modulates isoproterenol induced myocardial ischemia through antioxidant and anti-dyslipidemic effect in male Wistar rats. Biomed Pharmacother 87: 705-713, 2017.

25. Yaffe PB, Power Coombs MR, Doucette CD, Walsh M and Hoskin DW: Piperine, an alkaloid from black pepper, inhibits growth of human colon cancer cells via G1 arrest and apoptosis triggered by endoplasmic reticulum stress. Mol Carcinog 54: 1070-1085, 2015

26. Deng $\mathrm{T}$, Wang $\mathrm{Y}$, Wang $\mathrm{C}$ and Yan $\mathrm{H}$ : FABP4 silencing ameliorates hypoxia reoxygenation injury through the attenuation of endoplasmic reticulum stress-mediated apoptosis by activating PI3K/Akt pathway. Life Sci 224: 149-156, 2019.

This work is licensed under a Creative Commons Attribution-NonCommercial-NoDerivatives 4.0 International (CC BY-NC-ND 4.0) License. 